\title{
Smart Home System Design Based on Internet of Things
}

$$
\text { Ziyao Zou }{ }^{\mathrm{a}} \text {, Yan Wu, Weiguang Yang and Xin Wang }
$$

College of Information and Control Engineering Shenyang Jianzhu University, Shenyang 110168; China;

\author{
a455265987@qq.com
}

*The corresponding author

Keywords: The Internet of things; Smart home; Intelligent monitoring; ZigBee network

\begin{abstract}
With the rapid development of Internet technology and wide application, the smart home system based on Internet of things technology application was born. With services for the design scheme for drive, designed on the basis of the Internet of things technology of intelligent household design scheme, realize the household environment of intelligent monitoring and remote control, the scheme is designed to provide users with more intelligent and more comfortable service.
\end{abstract}

\section{Introduction}

With the continuous improvement of the quality of people's life, the living environment is needed more and more intelligent, automatic. Not only at home but abroad are developing the construction of the smart home. However, the study of Smart home still lack of integrity. We combine with the ZigBee technology and design a fully perceived, interconnected design of smart home in this article.

\section{Intelligent Household Overall Design}

The construction of the smart home is based on ubiquitous network. When users enter the smart home, intelligent control system environment take actions directly: smart home sensor module perceives the home environment and varies with the condition of monitoring the home environment to adjust the smart home devices. The system is hoped to achieve a comprehensive perception of the home environment and the effect of automatic hardware and software interconnection.

\section{(1) Air Conditioning Control.}

The HTU21D temperature and humidity sensors is chosen in this paper to collect the data of temperature and humidity of the smart home information, and then automatically drive and control the execution node in the smart home air conditioning, making the temperature and humidity in the most appropriate energy conservation and emissions reduction.

\section{(2) Lighting Control.}

The NHZD10CP light sensor is chosen in this paper to acquire light intensity information of smart home, and then automatically drive and control the brightness of light, making household light achieve optimum results.

(3) Air Quality Monitoring.

The TGS2600 air quality sensor, PM2.5 dust sensor is chosen in this paper to collect PM2.5 air quality information, $\mathrm{CO} 2$ and $\mathrm{O} 2$ concentration, and then automatically drive and control of air purification and ventilation equipment, making household always keep the air fresh.

\section{(4) Fire Monitoring.}

The MQ - 7 sensor is selected in the paper to collect CO concentration in intelligent household information, which is automatically drive buzzer alarm to forecast the effect of fire in time when more than a certain $\mathrm{CO}$ threshold.

\section{(5) Security Control.}

The infrared curtain sensor is adopted in this paper to sense the infrared radiation emitted by foreign personnel. When an emergency intrusion is encountered, the sensor immediately triggers an alarm to protect the user's life and property. 


\section{(6) SMS to Monitor.}

$3 \mathrm{G}$ module is adopted in this paper to make the remote user the remote user to achieve the monitoring of HEA. The design of using AT commands to drive EM560 $3 \mathrm{~g}$ module to achie ve the message transmission.

\section{The Smart Home Control System Implementation}

Smart home control system can be divided into sensing layer, transport layer, application layer. The sensing layer node deployed in the home is self-organizing through ZigBee protocol. The sensing layer communicates with various traditional network facilities through the Internet of Things gateway .Transport layer will collect the perception of gateway data and transport to the center of the cloud application server processing. The part of application in the center of the cloud layer of is able to carry out a series of operations such as cleaning, storing and analyzing the data. The application layer sends the control instruction to the device controller of the designated intelligent home and receives the specific device address of the device controller. Module is to send instructions to the adapter port. The fig. 1 shows the smart home system overall architecture diagram.

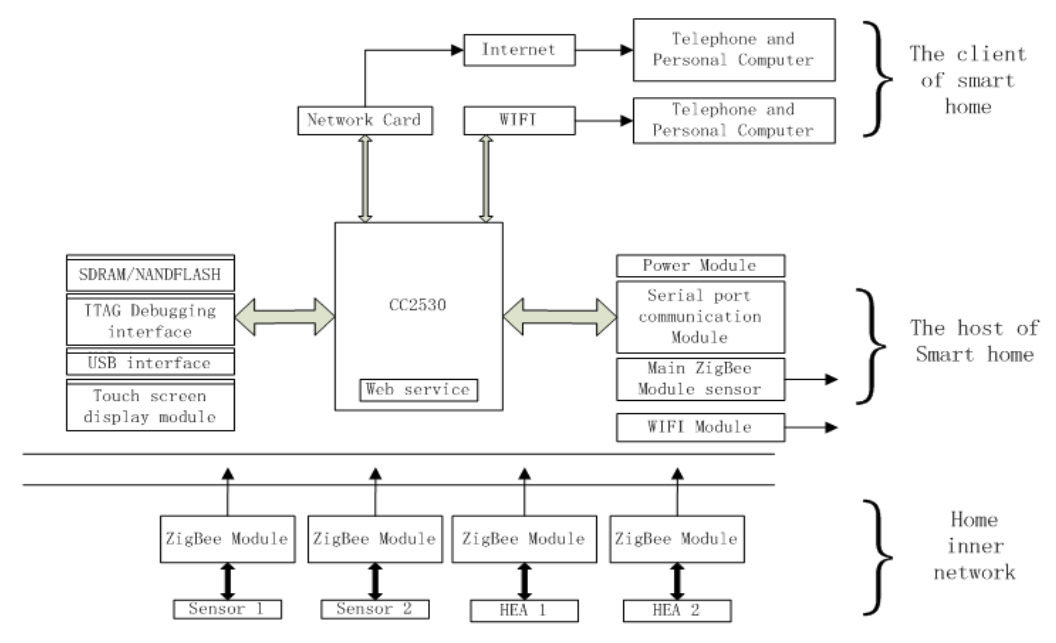

Figure1. Architecture of smart home system

\section{The Design of Hardware.}

1. Terminal Sensing Node.

The number of sensors in the smart home is determined by the scope of the furnishings [4]. The sensor is perceived as a node for intelligent home information. In addition to collecting intelligent home environment information, The sensor but also need to be able to carry out data transmission with the microcontroller. In this design, AM3358 based on Kernel Cortex-A 8 is chosen by microcontrollers and the Linux system for the JDK8 virtual machine environment is configured. JAVA is used to achieve data and information transmission, terminal implementation node control, and strive to simplify the terminal aware node deployment and development complexity. Meanwhile, I2C is selected to achieve the perception of information nodes and for the microprocessor data transmission and information exchange in the design.

2. The Design of Gateway.

The gateway in smart home is responsible for in addition to detecting and controlling the device but also connect with the Internet.

S5PV210 for the master chip development board x210ii is based on Cortex-A8 to achieve ZigBee gateway function in this design. CC2530 wireless receiver chip has a wealth of interface resources and support the Z-Stack protocol stack. Development board achieve and ZigBee network connection through the serial port to connect CC2530 chip. Development Platform based on x210ii 
Andrews background is easy to develop terminal programs, which can be transmitted to the embedded data sensor web server.

3. The Design of the Terminal Execution Node.

The ZigBee coordinator sends the aggregated perceptual data information to the background terminal through the serial port. The background terminal sends the control command from the serial port to the coordinator according to the analysis of the information. The coordinator performs the node control through the ZigBee terminal to control the action of all kinds of intelligent devices. Coordinator controls the actions of various types of intelligent devices through the ZigBee terminal.

The Design of Smart Home Networking. In the design of smart home, every node is essential to communicate with each other mutual perception. So the sensing node is essential to be set up as a local area network. ZigBee technology is used to establish self-organizing network [5]. It is usually initiated by the coordinating node in the local network. The coordinator and the routing node are responsible for sending and receiving data. The coordinator selects a network ID and a channel at first when the coordinating node initiates bureau and then open the entire topology network. ZigBee router also acts as a coordinator in this process, is also responsible for the network configuration.

The Design of Control Node Software. The main task of the control node software design is to analyze and feedback the received gateway commands. The workflow of the control node is that searching network after starting instruction, and then the network is added immediately after searching the network. The work process of control node is shown in Fig. 2.

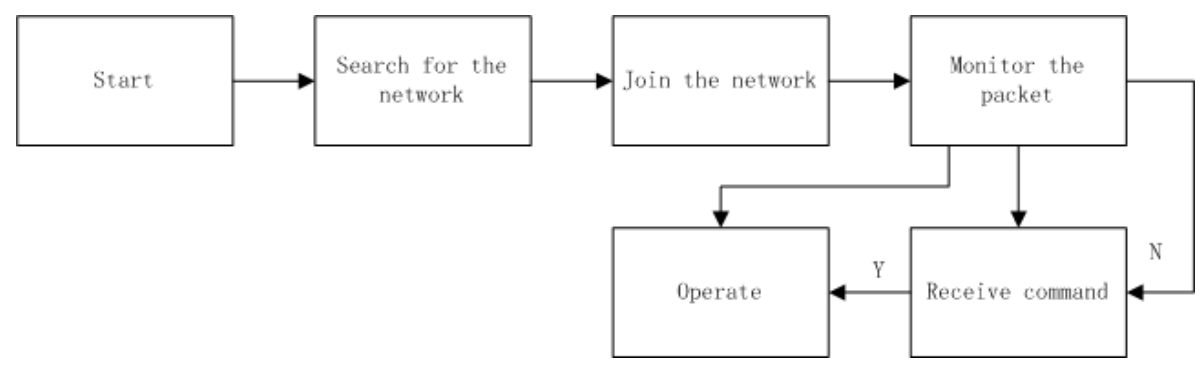

Figure 2. The work process of control node software

\section{Conclusion}

A smart home control system is proposed, which is based on the Internet of Things. The monitoring of HEA is achieved through the ZigBee wireless network, WIFI wireless network and mobile phone text messages in order to provide users with smart and comfortable living environment.

\section{Acknowledgments}

Project supported by the programs of college students ' innovation and entrepre- neurship (No.201610153037; 201610153084).

\section{Reference}

[1] PENG Junjie, He Hui, ZHU Pingan, LIU Yanping, ZHANG Xuejun, JIN Yi. Zigbee-based new approach to smart home [J]. Journal of Shanghai University (English Edition), 2010,01:12-16.

[2] QIN Haichun, ZHAO Cong, WU Bo, ZHANG Li. Nursing bed used in the smart home environment[J]. Computer Aided Drafting,Design and Manufacturing, 2013,01:58-61.

[3] S.M.T. Bathaee, A. Fereidunian, A. Khajeh Amiri Hagh, H. Heydari. Design and Implementation of a Novel HomePlug-Based Solution for Low Cost and High Performance Smart Home Networking [J]. Journal of Electronic Science and Technology, 2014, 01:33-38. 
[4] WANG Guang-wei, LU Sheng-li. Smart Home Gateway Based on ZigBee Technology [J]. International Journal of Plant Engineering and Management, 2015, 04: 240-249.

[5] Zou L, Chen L, Özsu M T.K-automorphism: a general framework for privacy preserving network publication[J]. Proceedings of the VLDB Endowment, 2009,2(1):946-957.

[6] Chen R, Philip S Y. Correlated network data publication via differential privacy[J].The International Journal on Very Large Data Bases,2014, 23(4): 653-676. 